\title{
HOMEOMORPHISMS WITH POLYHEDRAL IRREGULAR SETS. I
}

\author{
BY
}

\author{
P. F. DUVALL, JR. AND L. S. HUSCH( $\left.{ }^{1}\right)$
}

ABSTRACT. Homeomorphisms on open manifolds with polyhedral irregular sets are studied. For high dimensions, necessary and sufficient conditions for certain codimension three irregular sets to be tame are given. Several examples of homeomorphisms with wild irregular sets are given.

1. Introduction. A homeomorphism $b$ on a metric space $(X, d)$ is regular (positively regular) at $x$ if, for each $\epsilon>0$, there exists $\delta>0$ such that $d(x, y)<\delta$ implies that $d\left(b^{i}(x), b^{i}(y)\right)<\epsilon$ for each integer $i$ ( $\left.i>0\right)$. Two homeomorphisms, $b_{0}$ and $b_{1}$, on a space $X$ are topologically equivalent if there exists a homeomorphism $k$ of $X$ onto itself such that $b_{1}=k^{-1} b_{0} k$. B. V. Kerékjártó [22] showed that homeomorphisms on the 2 -sphere which are regular except possibly on a finite number of points were topologically equivalent to fractional linear transformations of complex numbers. S. Kinoshita ([24], [25]), T. Homma and S. Kinoshita [15], and L. Husch ([18], [19], [20]) extended these investigations into higher dimensions. S. Kaul [21] investigated homeomorphisms which fail to be regular on compact zero dimensional sets.

Suppose $M$ is an open manifold which is an open mapping cylinder neighborhood of some continuum $P \subseteq M$. By using the product structure of $M-P$, it is possible to define a homeomorphism $b$ of $M$ onto itself such that $b$ is regular everywhere except on $P$ and is positively regular everywhere. The metric on $M$ is to be the metric induced from the metric of the one point compactification of $M$, $M \cup\{\infty\}$. Note, in addition, the extension of $b$ to $M \cup\{\infty\}$ is not regular at $\infty$; we shall say " $b$ is not regular at $\infty$ ".

In this paper we investigate homeomorphisms of open manifolds $M$ which are regular except at $\infty$ and on a compact polyhedron $P$ and explore to what extent we obtain a converse to the above construction. Our main question is whether $P$ need be tamely embedded in $M$. The answer is no by Theorem 7.1. We define a property of continua, AFG, which provides a necessary and sufficient condition for certain $h, M$ and $P$ (Theorem 4.9 ) so that $P$ be tamely embedded.

Received by the editors June 7, 1971 and, in revised form, February 3, 1972 and August 15, 1972.

AMS (MOS) subject classifications (1970). Primary 57E99.

(1) Research of the second author was partially supported by NSF Grant GP-33872. 
In $\$ 2$ we have the preliminaires and in $\$ 3$ we define $A F G$ and develop the theory of continua having AFG. $\$ 4$ and $\$ 5$ contain the proof of the necessity and sufficiency of AFG in taming polyhedral sets. In $\$ 6$ we consider mapping cylinder neighborhoods and in $\$ 7$ we have several examples related to extending this theory.

2. Preliminaries. If $U$ is an open connected $n$-manifold with a finite number of ends [13], let $U_{\infty}=U \cup\{\infty\}$ be the one-point compactification of $U$. We note that, in general, the regularity of a homeomorphism depends upon the metric. However, if the space we are working with is compact, then regularity does not depend upon the metric. Hence when we consider open manifolds, we shall consider the metric induced from the one point compactification. We say that a homeomorphism is not regular at $\infty$ if the induced homeomorphism $b_{\infty}$ of $U_{\infty}$ is not regular at $\infty$. [If we do not assume that $U$ has a finite number of ends, then we can consider the Freudenthal end point compactification $U_{*}$ of $U$. If $b$ is a homeomorphism of $U$, then there is induced a unique homeomorphism $b_{*}$ of $U_{*}$. If $b_{*}$ is regular except on $U_{*}-U$, then it follows from [21] that $U$ has either one or two ends (see [11] for details). Hence there is no loss of generality in assuming that $U$ has a finite number of ends and we shall only consider such $U$ in this paper.]

Let $U$ be a connected open $n$-manifold and let $P$ be a $k$-dimensional continuum in $U, k+2 \leq n$.

Proposition 2.1. Let $b$ be a bomeomorphism of $U$ onto itself such that $b$ is regular on $U-P$ and is not regular on $P \cup\{\infty\}$. For each compact set $C \subseteq U-P$, either $\overline{\lim }_{i \rightarrow-\infty} b^{i}(C)=\infty$ and $\overline{\lim }_{i \rightarrow+\infty} b^{i}(C)$ is a nonempty subset of $P$ or $\overline{\lim }_{i \rightarrow+\infty} b^{i}(C)=\infty$ and $\overline{\lim }_{i \rightarrow-\infty} b^{i}(C)$ is a nonempty subset of $P$.

Proof. Let $U_{0}$ be the decomposition space obtained from $U_{\infty}$ by collapsing $P$ to a point and let $\pi: U_{\infty} \rightarrow U_{0}$ be the natural projection. Define $b_{0}: U_{0} \rightarrow U_{0}$ by $b_{0}(\pi(x))=\pi(b(x))$ for each $x \in U$ and $b_{0}(\pi(\infty))=\pi(\infty)$. Note that $b_{0}$ is a homeomorphism of $U_{0}$ onto itself. It is easily seen that $b_{0}$ is regular on $U_{0}$ $\{\pi(P), \pi(\infty)\}$. Since $h_{0}(\pi(P))=\pi(P)$ and $h_{0}(\pi(\infty))=\pi(\infty), h_{0}$ fails to be regular at $\pi(P)$ and $\pi(\infty)$ [16]. Hence, by [24], for each compact set $C \subseteq U_{0}-\{\pi(P), \pi(\infty)\}$, either $\overline{\lim }_{i \rightarrow-\infty} b_{0}^{i}(C)=\pi(\infty)$ and $\overline{\lim }_{i \rightarrow+\infty} b_{0}^{i}(C)=\pi(P)$ or $\overline{\lim }_{i \rightarrow+\infty} b_{0}^{i}(C)=\pi(\infty)$ and $\overline{\lim }_{i \rightarrow-\infty} b_{0}^{i}(C)=\pi(P)$. The result now follows easily.

Corollary 2.2. If for each compactum $C \subseteq U-P, \overline{\lim }_{i \rightarrow-\infty} b^{i}(C)=\infty$, then for each compactum $C \subseteq U, \overline{\lim }_{i \rightarrow+\infty} b^{i}(C) \subseteq P$.

It follows from the proof of Proposition 2.1 and [16] that if we assume that $P$ has a finite number of components then $P$ is connected.

For each $x \in U$, let $O(x)=\operatorname{cl}\left\{b^{i}(x)\right\}_{i=0}^{\infty}$ and $K(x)=\bigcap_{i=0}^{\infty} O\left(b^{i}(x)\right)$. Suppose $y, z \in O(x), y=\lim _{n \rightarrow+\infty} b^{i n}(x), z=\lim _{n \rightarrow+\infty} b^{j n}(x)$; define $y \cdot z=$ $\lim _{n \rightarrow+\infty} b^{i} n^{+i} n(x)$. The following was shown in [10]. 
Proposition 2.3. If $b$ is positively regular on $U$, then

(i) $O(x)$ is a topological semigroup with the multiplication defined as above;

(ii) $K(x)$ is an ideal in $O(x)$ and bence is a topological group;

(iii) if $y \in K(x)$, then $O(y)=K(x)$.

Corollary 2.4. If $b$ is positively regular on $U$ and $b \mid P$ is the identity, then for each $x \in U, \lim _{i \rightarrow+\infty} b^{i}(x)$ exists and the function $f(x)=\lim _{i \rightarrow+\infty} b^{i}(x)$ is $a$ retraction of $U$ to $P$.

Proof. Suppose $x \in U$ and let $z \in K(x)$. Hence $z=\lim _{i \rightarrow+\infty} b^{i n}(x)$; since $b(z)=z$, by 2.3 (iii), $K(x)=\{z\}$. It follows that $\lim _{i \rightarrow+\infty} b^{i(x)}$ exists. By Proposition 2.1 and positive regularity, $f(x) \in P$ for each $x \in U$. The continuity of $f$ follows from positive regularity.

Proposition 2.5. Let $U$ be a connected open $n$-manifold and let $P$ be a $k$ dimensional continuum in $U, k+2 \leq n$. Let $b$ be a bomeomorphism of $U$ onto itself which is regular except on $P \cup\{\infty\}, b \mid P$ is the identity and $b$ is positively regular everywhere. Then

(i) $P$ is an absolute neighborbood retract;

(ii) the inclusion $P \subseteq U$ is a bomotopy equivalence;

(iii) the natural projection $p$ of $U-P$ onto the orbit space $\hat{U}$ of $b \mid U-P$ is a covering map;

(iv) $\hat{U}$ is a closed n-manifold;

(v) $f$ induces a map $\hat{f}: \hat{U} \rightarrow P$ such that $\hat{f p}=f$.

Proof. (i) Follows from Proposition 2.4.

To show (ii), by $\left[35\right.$, p. 405], it suffices to show that the induced map $f_{\#}$ : $\pi_{i}(U) \rightarrow \pi_{i}(P)$ is one-to-one. Let $\lambda$ be a mapping of the $i$-sphere $S^{i}$ into $U$ such that $f \lambda$ is inessential. There exists $\epsilon>0$ such that if $d(g(x), f \lambda(x))<\epsilon$ for all $x \in S^{i}$, then $g$ and $f \lambda$ are homotopic. By Corollary 2.2 , there exists $j$ such that $d\left(b^{j} \lambda(x), f \lambda(x)\right)<\epsilon$ for all $x \in S^{i}$. Hence $\lambda$ is inessential.

(iii) and (iv) follow from [23] and [24]. We leave (v) for the reader.

We shall assume familiarity with either [17] or [40]. Let $f: N^{n} \rightarrow M^{m}$ be an embedding of a manifold into a manifold; $f$ is locally flat if for each $x \in N^{n}$, there exists a neighborhood $U$ of $f(x)$ in $M^{m}$ such that the pair $\left(U, U \cap f\left(N^{n}\right)\right.$ ) is homeomorphic to either $\left(R^{m}, R^{n}\right)$ or $\left(R^{m}, R_{+}^{n}\right)$ where $R^{i}$ is Euclidean $i$-space and $R_{+}^{i}$ is Euclidean half-i-space with usual inclusions. An embedding $f: P \rightarrow M$ of a polyhedron $P$ into a manifold $M$ is locally flat if for some triangulation $K$ of $P, f$ restricted to each open simplex in $K$ is locally flat. $f: P \rightarrow M$ is locally tame at $x \in P$ if there is a neighborhood $U$ of $p$ in $M$ and a homeomorphism $k$ of $\mathrm{cl}(U)$ onto a subpolyhedron in $M$ such that $k(\mathrm{cl}(U \cap f(P)))$ is a subpolyhedron. $f$ is locally tame if it is locally tame at each $x \in P$. 
3. Local homotopy properties for embedded continua. In this section, we study the 1-dimensional homology and homotopy of the local neighborhood system of a continuum embedded in an ENR, Euclidean neighborhood retract-i.e. a retract of some open subset of some finite dimensional Euclidean space. The notion of AFG defined below was suggested by the studies of UV properties in [3], [28], [29] and 1-ALG in [14]; some of our proofs are similar to those in the above papers.

Definition 3.1. Suppose that $A$ is a continuum embedded in the ENR $X$. We say that $A$ has property AFG if there is a neighborhood $W$ of $A$ such that if $U$ is any neighborhood of $A, U \subseteq W$, there is a neighborhood $V$ of $A$ such that $V \subseteq U$ and, whenever $f: S^{1} \rightarrow V$ is a map such that $f_{*}: H_{1}\left(S^{1}\right) \rightarrow H_{1}(U)$ is the zero map, then $f$ extends to a map of the disk into $U$.

We first show that AFG is a topological property. The following lemma is a result of standard local homotopy properties of ENR's.

Lemma 3.2. Let $X$ be an ENR, $A \subseteq X$, a continuum. Let $W$ be a neighborbood of $A$ and $f: W \rightarrow X$ a map such that $f \mid A \sim 1_{A}$ (" $\sim$ " means "is bomotopic to"). Then for each neighborbood $U$ of $A$ there is a neighborbood $V$ of $A$ such that $f: V \rightarrow U$ is bomotopic to the inclusion $V \subseteq U$.

Theorem 3.3. Suppose that $A$ and $B$ are continua embedded in the ENR's $X$ and $Y$ respectively, and suppose that $A$ dominates $B$. If $A$ bas the property $\mathrm{AFG}$, then $B$ has the property AFG.

Proof. Let $\rho: A \rightarrow B$ and $\eta: B \rightarrow A$ be maps such that $\rho \eta \sim 1_{B}$. By the neighborhood extension properties of ENR's [4], we may extend $\eta$ to $\eta^{*}: W_{0} \rightarrow$ $X$, where $W_{0}$ is a neighborhood of $B$. Let $W$ be the neighborhood of $A$ promised by AFG and let $W_{1}$ be $\eta^{*-1}(W)$. Extend $\rho$ to $\rho^{*}: W_{2} \rightarrow W_{1}$, where $W_{2}$ is a neighborhood of $A$ in $W$. Let $U$ be a neighborhood, $B \subseteq U \subseteq W_{1}$, and let $U_{1}=$ $\rho^{*-1}(U)$. Let $V_{1} \subseteq U_{1}$ be a neighborhood of $A$ promised by $A F G$, and let $V_{0} \subseteq U$ be a neighborhood of $B$ promised by 3.2 for $U$ and $\rho^{*} \eta^{*}$. Finally, let $V_{2}=V_{1} \cap \rho^{*-1}\left(V_{0}\right)$ and let $V=V_{0} \cap \eta^{*-1}\left(V_{2}\right)$. Suppose that $f: S^{1} \rightarrow V$ is a map such that $f_{*}: H_{1}\left(S^{1}\right) \rightarrow H_{1}(U)$ is the zero map. It follows that $\eta^{*} f$ extends to a map of the disk into $U_{1}$ so $\rho^{*} \eta^{*} f$ extends to a map of the disk into $U$. But $\rho^{*} \eta^{*} f \sim f$ in $U$ and the proof is complete.

Corollary 3.4. For continua, the AFG property depends only on bomotopy type.

Proposition 3.5. If $A$ bas the bomotopy type of a finite complex, $A$ bas property AFG if and only if $\pi_{1}(A)$ is abelian. 
Proof. By 3.3, we may assume that $A$ is a subcomplex of some Euclidean space. If $\pi_{1}(A)$ is abelian, let $W$ be the interior of a regular neighborhood of $A$. If $U \subseteq W$ is any neighborhood of $A$, let $V$ be the interior of a regular neighborhood of $A, V \subseteq U \subseteq W$. If $f: S^{1} \rightarrow V$ is null homologous in $U$, it is null homologous and thus null homotopic in $W$, since $\pi_{1}(W) \cong \pi_{1}(A) \cong H_{1}(W)$.

If $\pi_{1}(A)$ is not abelian, let $f: S^{1} \rightarrow A$ represent a nontrivial commutator in $\pi_{1}(A)$. Then $f$ is null homologous but not null homotopic in every regular neighborhood of $A$, so that $A$ cannot have property AFG.

Remark. 3.5 is not true for arbitrary $A$. For example, if $A$ is the $\sin x^{-1}$ circle with a simple closed curve attached at a point, $\pi_{1}(A) \cong Z$, But $A$ does not have AFG.

Suppose that $A$ is a continuum in the $\operatorname{ENR} X$, then the collection $\{U \mid U$ is a a neighborhood of $A\}$ is directed by inclusion, and we have the corresponding inverse system $\left\{H_{1}(U) ; i_{*}\right\}$ where the $H_{1}(U)$ are the singular homology groups with $Z$ coefficients and the bonding maps are induced by inclusion. We let $\breve{H}_{1}(A)$ be the inverse limit of this system. Similarly, choosing a base point $* \in A$, we define $\check{\pi}_{1}(A, *)$ to be the inverse limit of groups $\pi_{1}(U, *)$.

Proposition 3.6. If $X$ is an ENR, and $A \subseteq X$ is a continuum with $A F G$, then $\check{\pi}_{1}(A, *)$ is abelian.

Proof. Let $A \subseteq \cdots \subseteq U_{n+1} \subseteq U_{n} \subseteq \cdots$ be a cofinal sequence of neighborhoods of $A$ in $X$. The Hurewicz map $b_{n}: \pi_{1}\left(U_{n}, *\right) \rightarrow H_{1}\left(U_{n}\right)$ gives a commutative diagram

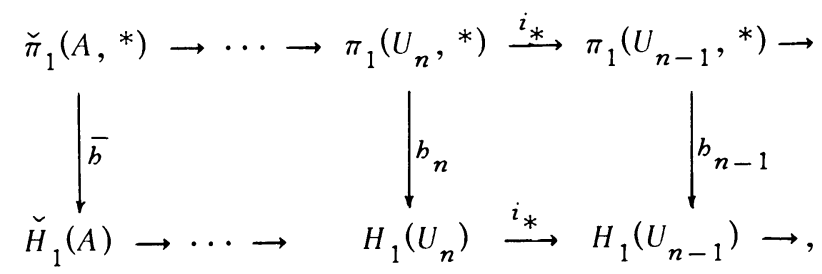

where $\bar{b}$ is the inverse limit of the $b_{n}$. If we choose the $U_{n}$ so that $U_{1}=W$ and $U_{n+1}$ is the $V$ guaranteed for $U_{n}$ in the definition of AFG, it follows that $b_{n}{ }_{n}$ /image $\left(i_{*}\right)$ is monic, which implies that $\bar{b}$ is monic, so that $\breve{\pi}(A, *)$ embeds in the abelian group $\breve{H}_{1}(A)$.

Remark. 3.6 is false if $\check{\pi}_{1}$ is replaced by $\pi_{1}$. Let $A_{0}$ be the $\sin x^{-1}$ curve union an isolated point in $R^{2}$, and let $A \subseteq R^{3}$ be the suspension of $A_{0}$. Then $A$ is AFG, but $\pi_{1}(A)$ is free on two generators.

Recall that an inverse limit system is said to be essentially constant if it has a cofinal subsequence $\cdots \rightarrow G_{3} \stackrel{f_{2}}{\rightarrow} G_{2} \stackrel{f_{1}}{\rightarrow} G_{1}$ such that $f_{i}$ : image $\left(f_{i+1}\right) \rightarrow$ image $\left(f_{i}\right)$ is an isomorphism for each $i$. For such a system, the projection of the 
inverse limit onto image $\left(f_{i}\right)$ is an isomorphism for each $i$. We will need the following proposition in the next section.

Proposition 3.7. Suppose that $A$ is AFG, $A$ is embedded in the ENR $X,\left\{U_{a}\right\}$ is the collection of neighborboods of $A$, and $* \in A$. If $\left\{H_{i}\left(U_{a}\right)\right\}$ is essentially constant, then $\left\{\pi_{1}\left(U_{a}, *\right)\right\}$ is essentially constant, and $\bar{b}: \check{\pi}_{1}(A, *) \rightarrow \check{H}_{1}(A)$ is an isomorphism.

Proof. Let $U_{1} \supseteq U_{2} \supseteq \cdots$ be a cofinal sequence of neighborhoods of $A$ guaranteed by the definition of essentially constant for $\left\{H_{1}\left(U_{a}\right)\right\}$. We may assume that the $U_{i}$ 's are chosen as in the proof of 3.6. Then we have

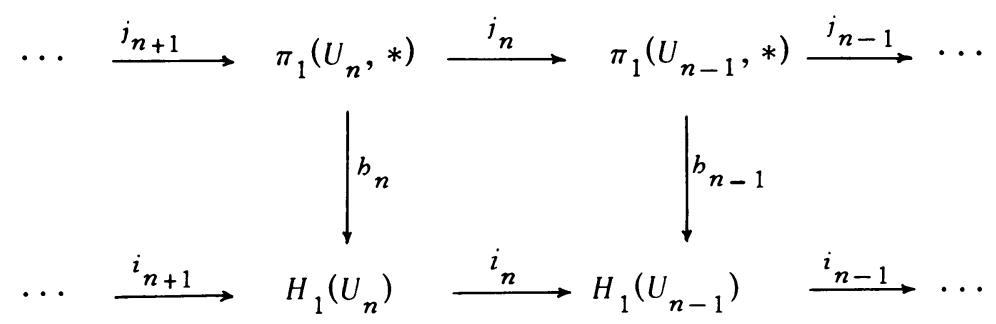

where $b_{n}$ is the Hurewicz map and $i_{n}, j_{n}$ are induced by inclusion. As before, $b_{n}$ | image $\left(j_{n+1}\right)$ is monic, and since $b_{n} j_{n+1}=i_{n+1} b_{n+1}$ and $b_{n+1}$ is onto, $b_{n}$ (image $\left.j_{n+1}\right)=$ image $i_{n+1}$. Therefore we have

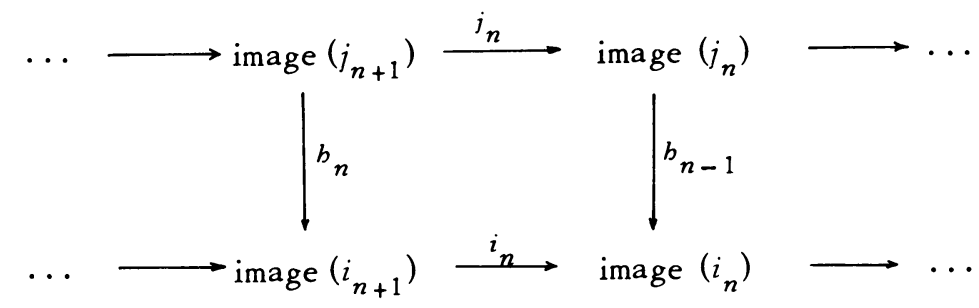

where $b_{n}, b_{n-1}, i_{n}$ are isomorphisms. The proposition follows.

Proposition 3.8. With the same notation as above, if $A$ is an ENR, then $\left\{\pi_{1}\left(U_{\alpha}, *\right)\right\}$ is essentially constant and $\check{\pi}_{1}(A, *) \simeq \pi_{1}(A, *)$.

Proof. As before choose a cofinal sequence $U_{1} \supseteq U_{2} \supseteq \ldots$ By standard properties of ENR's we may choose the $U_{i}$ 's so that there is a retraction $\rho$ : $U_{1} \rightarrow A$ and the diagram

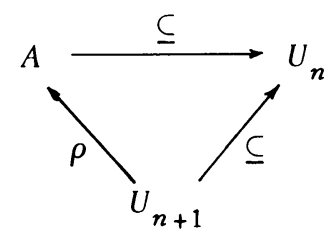


is homotopy commutative. Thus we have a diagram

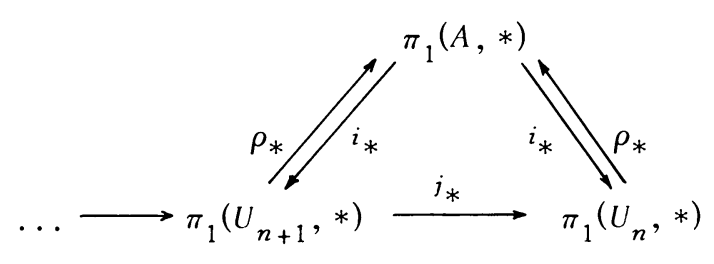

where $\rho_{*}$ is induced by $\rho$ and $i_{*}, j_{*}$ are induced by inclusion, $\rho_{*} i_{*}=1, i_{*} \rho_{*}=j_{*}$. A straightforward diagram chase completes the proof.

4. Sufficiency of AFG. Let $U$ be an open connected $n$-manifold, let $b$ be a homeomorphism of $U$ onto itself such that $b$ is regular except at $\infty$ and, on a compact polyhedron $P$ of dimension $k \leq n-3, b$ is positively regular everywhere and $b \mid P$ is the identity. Let $f: U \rightarrow P$ be the retraction given in Corollary 2.4 , let $q: U-P \rightarrow X$ be the natural projection onto the orbit space of $b \mid U-P$ and let $\hat{f}: X \rightarrow P$ be the map induced from $f$.

If $G$ is an Abelian group and $K$ is a group of operators of $G$ generated by $k$, let

$$
G_{K}=G /\{(1-k) g \mid g \in G\}, \quad G^{K}=\{g \in G \mid k(g)=g\} .
$$

Proposition 4.1. If $p \in P$, let $\left\{U_{i}\right\}_{i=1}^{\infty}$ be a neighborbood basis at $p$ of contractible open sets in $P$ such that $U_{i+1} \subseteq U_{i}$ for all $i$. Then $\left\{H_{1}\left(\hat{f}^{-1} U_{i}\right)\right\}$ is es. sentially constant and $\breve{H}_{1}\left(\hat{f}^{-1} p\right)$ is isomorpbic to the integers.

Proof. Let $Z$ be the group generated by the map induced by $b \mid f^{-1} U_{i}-U_{i}$ on $H_{1}\left(f^{-1} U_{i}-U_{i}\right)$. From Serre [32] we get a short exact sequence

$$
0 \rightarrow H_{1}\left(f^{-1} U_{i}-U_{i}\right)_{Z} \rightarrow H_{1}\left(\hat{f}^{-1} U_{i}\right) \rightarrow H_{0}\left(f^{-1} U_{i}-U_{i}\right)^{Z} \rightarrow 0
$$

It is easily seen that the inclusion maps induce a commutative diagram

$$
\begin{aligned}
& 0 \rightarrow H_{1}\left(f^{-1} U_{i}-U_{i}\right)_{Z} \rightarrow H_{1}\left(\hat{f}^{-1} U_{i}\right) \rightarrow H_{0}\left(f^{-1} U_{i}-U_{i}\right)^{Z} \rightarrow 0 \\
& 0 \rightarrow H_{1}\left(f^{-1} U_{i+1}-U_{i+1}\right)_{Z} \rightarrow H_{1}\left(\hat{f}^{-1} U_{i+1}\right) \rightarrow H_{0}\left(f^{-1} U_{i+1}-U_{i+1}\right)^{Z} \rightarrow 0
\end{aligned}
$$

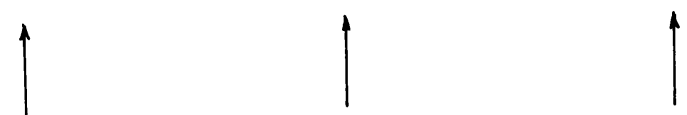

Since $f^{-1} U_{i}$ is contractible, $H_{1}\left(f^{-1} U_{i}-U_{i}\right) \cong H_{2}\left(f^{-1} U_{i}, f^{-1} U_{i}-U_{i}\right)$. By [35, p. 342], $H_{2}\left(f^{-1} U_{i}, f^{-1} U_{i}-U_{i}\right) \cong \bar{H}_{c}^{n-2}\left(U_{i}\right)$ and, by $\left[35\right.$, p. 340], $\bar{H}_{c}^{n-2}\left(U_{i}\right) \cong$ $H_{c}^{n-2}\left(U_{i}\right) \cong 0$. Hence $H_{1}\left(f^{-1} U_{i}-U_{i}\right) \cong 0$ and $H_{1}\left(f^{-1} U_{i}-U_{i}\right)_{Z} \cong 0$.

Note that if $M$ is a connected orientable manifold and $b$ is an orientationpreserving homeomorphism of $M$ onto itself, $b$ induces the identity map on $H_{0}(M)$; hence 


$$
H_{0}\left(f^{-1} U_{i}-U_{i}\right)^{Z} \cong H_{0}\left(f^{-1} U_{i}-U_{i}\right) .
$$

From the above diagram it follows that inclusion induces an isomorphism $H_{1}\left(\hat{f}^{-1} U_{i+1}\right) \cong H_{1}\left(\hat{f}^{-1} U_{i}\right)$.

Proposition 4.2. For each $i$, the inclusion induces one-to-one bomomorphisms $H_{1}\left(\hat{f}^{-1} U_{i}\right) \rightarrow H_{1}(X)$.

Proof. The result follows from the following commutative diagram

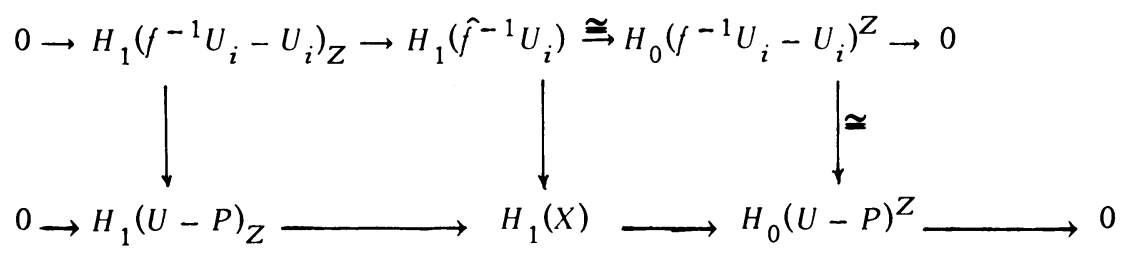

Proposition 4.3. Suppose $\hat{f}^{-1}(p)$ has AFG for some $p \in P$; then there exists a neighborbood basis at $p$ of contractible open sets $\left\{U_{i}\right\}_{i=1}^{\infty}$ in $P$ such that $U_{i+1} \subseteq U_{i}$ for all $i$ and, if $\sigma$ is a loop in $\hat{f}^{-1} U_{i+1}$ such that $\sigma$ is bomologous to zero in $\hat{f}^{-1} U_{i}$, then $\sigma$ is homotopically trivial in $\hat{f}^{-1} U_{i}$.

Proof. Let $\left\{U_{i}\right\}_{i=1}^{\infty}$ be a neighborhood basis at $p$ of contractible open sets in $P$ such that $U_{i+1} \subseteq U_{i}$ for all $i$. Given $U_{i}$ there exists an open set $V$ such that $\hat{f}^{-1}(p) \subseteq V \subseteq \hat{f}^{-1} U_{i}$ and if $\sigma$ is a loop in $V$ such that $\sigma$ is homologous to zero in $\hat{f}^{-1} U_{i}$, then $\sigma$ is homotopically trivial in $\hat{f}^{-1} U_{i}$, It follows from usual compactness arguments that there exists $j$ such that $\hat{f}^{-1} U_{j} \subseteq V$. The desired sequence is a subsequence of $\left\{U_{i}\right\}_{i=1}^{\infty}$.

Proposition 4.4. Suppose $\hat{f}^{-1}(p)$ bas AFG for some $p \in P$ and let $\left\{U_{i}\right\}_{i=1}^{\infty}$ be as in Proposition 4.3. Then for each $i$, the inclusion map induces the zero bomomorphism $\pi_{1}\left(f^{-1} U_{i+1}-U_{i+1}\right) \rightarrow \pi_{1}\left(f^{-1} U_{i}-U_{i}\right)$.

Proof. Consider the following commutative diagram:

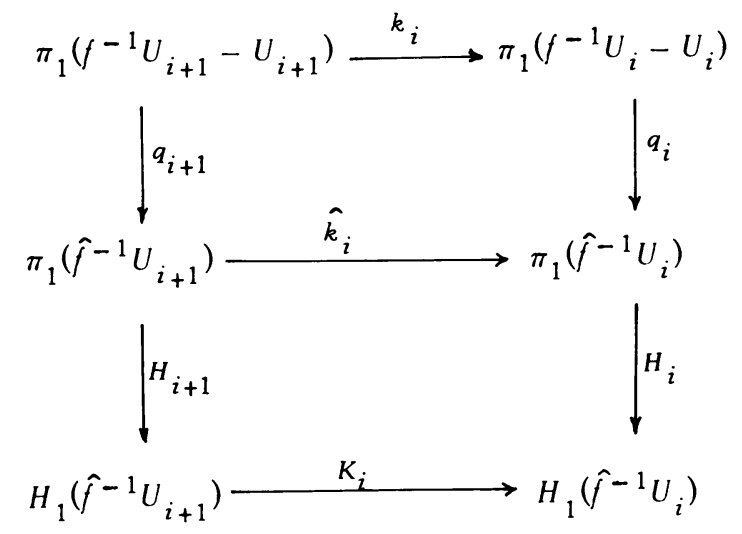


where $k_{i}, \hat{k}_{i}, K_{i}$ are induced by inclusions, $q_{i}=q \mid f^{-1} U_{i}-U_{i}$, and $H_{i}$ are the Hurewicz homomorphisms.

Suppose $\sigma \in \pi_{1}\left(f^{-1} U_{i+1}-U_{i+1}\right)$; since $H_{1}\left(f^{-1} U_{i}-U_{i}\right)=0, H_{1} q_{i} k_{i}(\sigma)=0$. $H_{1} \hat{k}_{i} q_{i+1}(\sigma)=0$ implies, by the previous proposition, that $\hat{k}_{i} q_{i+1}(\sigma)=0$. Since $q_{i}$ is one-to-one, $k_{i}(\sigma)$ is homotopically trivial.

Proposition 4.5. Suppose $B_{1} \subseteq B_{2}$ are open subsets of $P$; then $f_{*}$ : $\pi_{1}\left(f^{-1}\left(B_{2}\right)-B_{2}, f^{-1}\left(B_{1}\right)-B_{1}\right) \rightarrow \pi_{1}\left(B_{2}, B_{1}\right)$ is a bijection.

Proof. Suppose $\lambda:[0,1] \rightarrow B_{2}$ represents an element of $\pi_{1}\left(B_{2}, B_{1}\right)$; $\lambda(\{0,1\}) \subseteq B_{1}$. Cover $\lambda([0,1])$ by a finite number of contractible open sets $V_{1}$, $\cdots, V_{r}$ such that each $V_{i} \subseteq B_{2}$. Let $K$ be a simplicial complex such that $|K|=$ $[0,1]$ and $\sigma \in K$ implies $\lambda(\sigma) \subseteq V_{i}$ for some $i$.

If $\sigma$ is a 0 -simplex, let $\lambda^{\prime}(\sigma)$ be any point in $f^{-1}(\lambda(\sigma))-\lambda(\sigma)$. If $\sigma$ is a 1 simplex and bdry $\sigma=\left\{\sigma_{1}, \sigma_{2}\right\}$, define $\lambda^{\prime}: \sigma \rightarrow f^{-1} V_{i}-V_{i}$ to be any path from $\lambda^{\prime}\left(\sigma_{1}\right)$ to $\lambda^{\prime}\left(\sigma_{2}\right)$ where $\lambda(\sigma) \subseteq V_{i}$. Clearly $p \lambda^{\prime}$ and $\lambda$ represent the same elements of $\pi_{1}\left(B_{2}, B_{1}\right)$. Hence $f_{*}$ is onto.

Suppose $\lambda_{i}:[0,1] \rightarrow f^{-1}\left(B_{2}\right)-B_{2}$ such that $\lambda_{i}(\{0,1\}) \subseteq f^{-1}\left(B_{1}\right)-B_{1}, i=$ 0,1 , and suppose there exists $\Lambda:[0,1]^{2} \rightarrow B_{2}$ such that $\Lambda(x, i)=q p_{i}(x)$ and $\Lambda(i, t) \in B_{1}, i=0,1 ; x, t \in[0,1]$. Cover $\Lambda\left([0,1]^{2}\right)$ by a finite number of contractible open sets $V_{1}, \ldots, V_{r}, \ldots, V_{s}$ such that $V_{1}, \ldots, V_{r}$ cover $\Lambda(\{0,1\} \times[0,1])$ and $V_{i} \subseteq B_{1}, i=1, \ldots, r$, and $V_{i} \subseteq B_{2}, i=1, \ldots, s$. We may assume that $V_{i} \subseteq V_{i}^{\prime} \subseteq B_{2}$ where $V_{i}^{\prime}$ is contractible and each loop in $f^{-1} V_{i}-V_{i}$ is homotopically trivial in $f^{-1} V_{i}^{\prime}-V_{i}^{\prime}$.

Let $K$ be a simplicial complex such that $|K|=[0,1]^{2}, K$ contains two subcomplexes $L_{0}, L_{1}$ such that $\left|L_{i}\right|=[0,1] \times\{i\}, i=0,1$, and $\sigma \in K$ implies $\Lambda(\sigma)$ $\subseteq V_{i}$ for some $i$. If $\sigma$ is a 0 -simplex in $K-\left(L_{1} \cup L_{2}\right)$, choose $\Lambda^{\prime}(\sigma) \epsilon$ $f^{-1}(\Lambda(\sigma))-\Lambda(\sigma)$; if $\sigma$ is a 0 -simplex in $L_{i}$, choose $\Lambda^{\prime}(\sigma)=\lambda_{i}(\sigma)$. If $\sigma$ is a 1 simplex in $K-\left(L_{1} \cup L_{2}\right)$, let $W$ be the component which contains $\Lambda(\sigma)$ of the intersection of all $V_{i}$ 's which contain $\Lambda(\sigma)$. Define $\Lambda^{\prime}(\sigma)$ to be any path in $f^{-1} W-W$ which agrees with $\Lambda^{\prime}($ bdry $\sigma)$. Note that if $\sigma \in\{0,1\} \times[0,1], \Lambda^{\prime}(\sigma) \subseteq$ $f^{-1} B_{1}-B_{1}$. If $\sigma$ is a 1-simplex in $L_{i}$, let $\Lambda^{\prime}(\sigma)=\lambda_{i}(\sigma)$. Let $\sigma$ be a 2-simplex in $K$ and suppose $\Lambda(\sigma) \subseteq V_{i}$. Hence $\Lambda^{\prime}$ (bdry $\left.\sigma\right) \subseteq f^{-1} V_{i}-V_{i}$ and by hypothesis $\Lambda^{\prime}$ (bdry $\sigma$ ) is homotopically trivial in $f^{-1} V_{i}^{\prime}-V_{i}^{\prime}$. Hence we can extend $\Lambda^{\prime}$ to $\sigma$. Therefore $\lambda_{1}$ and $\lambda_{2}$ represent the same element of $\pi_{1}\left(f^{-1} B_{2}-B_{2}, f^{-1} B_{1}-B_{1}\right)$ and $f_{*}$ is one-to-one.

Theorem 4.6. If $\hat{f}$ is AFG (i.e. $\hat{f}^{-1}(p)$ bas AFG for all $p \in P$ ) and $B$ is an open connected subset of $P$, then there exists for each $p \in B$ an exact sequence

$$
1 \rightarrow \check{\pi}_{1} \hat{f}^{-1}(p) \rightarrow \pi_{1}\left(\hat{f}^{-1}(B)\right) \stackrel{\hat{f}^{*}}{\rightarrow} \pi_{1} B \rightarrow 1 .
$$


Proof. Let $\left\{U_{i}\right\}_{i=1}^{\infty}$ be as in Proposition 4.3 except that $U_{1} \subseteq B$. From the homotopy sequence of the pair $\left(\hat{f}^{-1} B, \hat{f}^{-1} U_{i}\right)$, we get an exact sequence

$$
\pi_{1} \hat{f}^{-1} U_{i} \stackrel{l_{i}}{\longrightarrow} \pi_{1} \hat{f}^{-1} B \stackrel{a}{\longrightarrow} \pi_{1}\left(\hat{f}^{-1} B, \hat{f}^{-1} U_{i}\right) \rightarrow 1 .
$$

By Proposition 4.1, $\left\{H_{1}\left(\hat{f}^{-1} U_{i}\right)\right\}$ is essentially constant; by Proposition 3.7, $\left\{\pi_{1}\left(\hat{f}^{-1} U_{i}\right)\right\}$ is essentially constant and hence, for $i>1$, image $l_{i}=$ image $\left\{j: \pi_{1}\left(\hat{f}^{-1}(p)\right) \rightarrow \pi_{1} \hat{f}^{-1}(B)\right\}$.

By Proposition 4.2, inclusion induces a one-to-one map $\check{H}_{1} \hat{f}^{-1}(p) \rightarrow H_{1} \hat{f}^{-1}(B)$. Hence by Proposition 3.7, $j$ is one-to-one.

We have the following commutative diagram
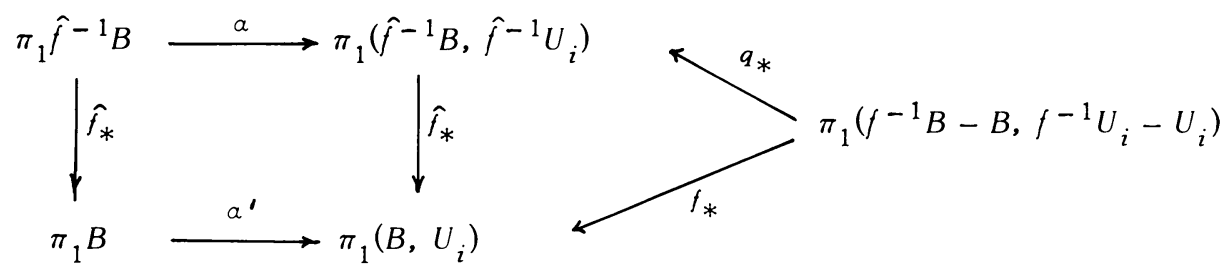

Since $U_{i}$ is contractible, the map $\alpha^{\prime}$, induced by inclusion, is an isomorphism. By Proposition 4.5, $f_{*}$ is an isomorphism and, from [35], $q_{*}$ is an isomorphism; hence $f_{*} \mid \pi_{1}\left(\hat{f}^{-1} B, \hat{f}^{-1} U_{i}\right)$ is an isomorphism. It follows that the kernel of $f_{*} \mid \pi_{1} \hat{f}^{-1} B=$ kernel of $\alpha$ and $\hat{f}_{*} \mid \pi_{1} \hat{f}^{-1} B$ is onto.

Theorem 4.7. If $\hat{f}$ is AFG, $p \in P, B$ is the open star of $p$ in some triangulation of $P$ such that each component of $B-\{p\}$ is simply connected, then $f^{-1} B$ is bomeomorphic to the interior of a compact manifold provided $n \geq 6$.

Proof. Let us first consider the case when $B-\{p\}$ is connected. Let $B=$ $\bigcup_{i=1}^{\infty} B_{i}$, where $B_{i}$ is the closed star of $p$ in some triangulation (compatible with the triangulation in the hypothesis) of $P, B_{i} \subseteq$ int $B_{i+1}$. By Proposition 3.7, $\check{\pi}_{1} \hat{f}^{-1}(p) \rightarrow \breve{H}_{1} \hat{f}^{-1}(p)$ is an isomorphism and, from Proposition 4.1, $\check{\pi}_{1} \hat{f}^{-1}(p)$ is isomorphic to the integers. By Theorem 4.6, for each $i, \pi_{1} \hat{f}^{-1}\left(B-B_{i}\right)$ is isomorphic to the integers and the inclusion maps induce isomorphisms

$$
\check{\pi}_{1} \hat{f}^{-1}(B) \cong \pi_{1} \hat{f}^{-1}\left(B-B_{1}\right) \cong \pi_{1} \hat{f}^{-1}\left(B-B_{2}\right) \cong \ldots
$$

Note that $\hat{f}^{-1}(B)$ has one end $\epsilon$ and, in the terminology of Siebenmann, $f_{1}$ is stable at $\epsilon$ [33] (essentially constant at $\epsilon$ [34]) and $\pi_{1}(\epsilon) \cong$ integers.

We will now show that the end $\epsilon$ is tame [33]; i.e. $\hat{f}^{-1}\left(B-B_{i}\right)$ is dominated by a finite complex $[33$, p. 23]. By $[35$, p. 342],

$$
H_{j}\left(f^{-1}\left(B-B_{i}\right), f^{-1}\left(B-B_{i}\right)-\left(B-B_{i}\right)\right) \cong \bar{H}_{c}^{n-j}\left(B-B_{i}\right) .
$$

By [35, p. 322], $\bar{H}_{c}^{n-j}\left(B-B_{i}\right) \cong \widetilde{H}^{q}\left(\left(B-B_{i}\right)^{+}\right)$, where $\left(B-B_{i}\right)^{+}$is the one-point compactification of $B-B_{i}$. But $B-B_{i}$ is homeomorphic to the product of a 
compact polyhedron with the real numbers. Hence $\widetilde{H} q\left(\left(B-B_{i}\right)^{+}\right)$is finitely generated and hence the homology of the pair $\left(f^{-1}\left(B-B_{i}\right), f^{-1}\left(B-B_{i}\right)-\left(B-B_{i}\right)\right)$ is finitely generated, and since the homology of $f^{-1}\left(B-B_{i}\right)$ is finitely generated, the homology of $f^{-1}\left(B-B_{i}\right)-\left(B-B_{i}\right)$ is finitely generated.

Since $q \mid f^{-1}\left(B-B_{i}\right)-\left(B-B_{i}\right)$ is an inf inite cyclic covering space and $\pi_{1} \hat{f}^{-1}\left(B-B_{i}\right)$ $\cong$ integers, it follows that $f^{-1}\left(B-B_{i}\right)-\left(B-B_{i}\right)$ is simply connected. Hence $\hat{f}^{-1}\left(B-B_{i}\right)$ satisfies condition NF $k$ of Wall [37, p. 61] for all $k \geq 2$ and, by Theorem $\mathrm{B}$ of [37], satisfies condition $\mathrm{F} k$ for all $k \geq 2$. By Theorem $\mathrm{E}$ of [37], $\hat{f}^{-1}\left(B-B_{i}\right)$ satisfies condition $D k$ for some $k$ (for example, $k \geq 2 n+2$ ). Hence by Theorem $\mathrm{F}$ of [37], $f^{-1}\left(B-B_{i}\right)$ is dominated by a finite complex (in fact, has the homotopy type of a finite complex).

The theorem now follows from [33] (although the results in [33] are stated for differentiable manifold, they are also valid for topological manifolds, see [38]).

If $B-\{p\}$ is not connected, we consider each end of $B$ separately.

Corollary 4.8. If $\hat{f}$ is AFG and $P$ is a manifold of dimension $k \neq 2$ (not necessarily triangulable), then for each open $k$-cell $B$ in $P, \hat{f}^{-1}(B)$ is bomeomorpbic to the interior of a compact manifold provided $n \geq 6$.

Theorem 4.9. Let $U$ be an open connected $n$-manifold, $n \geq 6$, let $b$ be $a$ bomeomorphism of $U$ onto itself such that $b$ is regular except at $\infty$ and on $a$ compact polybedron $P$ of dimension $k \leq n-3, b$ is positively regular on all of $U$ and $b \mid P$ is the identity. If the components of the link of each point in $P$ in some triangulation of $P$ are simply connected and if $\hat{f}$, the map induced on the orbit space of $b \mid U-P$ to $P$, is AFG, then $P$ is locally flat in $U$.

[Note that this theorem is not as general as the one stated in [41]; we had found an error in an earlier version of this paper since [41] appeared.]

Proof. We will show that $U-P$ is $1-\mathrm{LC}$ at each point of $P$, i.e. if $p \in P$ and $V_{1}$ is an open subset of $U$ containing $p$, then there exists an open subset $V_{2}$ of $U$ such that $p \in V_{2}$ and each mapping of $S^{1}$ into $V_{2}-P$ is null-homotopic in $V_{1}-P$. Let $V_{1}$ be given. Let $B$ be the open star of $p$ in some triangulation of $P$ such that $B \subseteq V_{1}$. By Corollary 4.8, $\hat{f}^{-1} B$ is homeomorphic to the interior of a compact manifold. Let $B_{0}$ be a codimension zero compact submanifold (with locally flat boundary) of $\hat{f}^{-1} B$ which is a deformation retract of $\hat{f}^{-1} B$. From obstruction theory it follows that there exists a map $g: B_{0} \rightarrow S^{1}$ such that $g$ induces an isomorphism of fundamental groups. Let $s \in S^{1}$ and homotope $g$ to a map $g^{\prime}$ such that $g^{\prime}$ is transverse regular with respect to $s$ $\left[38\right.$, p. 245]. Hence $\left(g^{\prime}\right)^{-1}(s)$ is a codimension one submanifold of $B_{0}$. Since $\pi_{1}$ bdry $B_{0} \rightarrow \pi_{1} B_{0}$ is an isomorphism, $\left(g^{\prime}\right)^{-1}(s) \cap$ bdry $B_{0}$ can be assumed to 
be a submanifold. Pick a component $B_{1}$ of $\left(g^{\prime}\right)^{-1}(s)$ such that each component $B_{2}$ of $q^{-1} B_{1}$ separates $q^{-1} B_{0}$. We can perform surgery on $B_{2}$ so that we can assume that $\pi_{1} B_{2}$ is trivial and hence each component of $q^{-1} B_{0}-B_{2}$ is simply connected ([38], [5]). Let $B_{3}$ be the closure (in $U$ ) of the component of $q^{-1} B_{0}-$ $B_{2}$ whose closure intersects $P$. There exists by Proposition 2.1 an integer $k$ such that $b^{k}\left(B_{3}\right) \subseteq V_{1}$. Let $V_{2}=$ int $b^{k}\left(B_{3}\right)$ and note that $V_{2}-P$ is simply connected and hence $U-P$ is 1-LC at each point of $P$.

Let $K$ be a triangulation of $P$ such that each each simplex in $K$ lies in an open $n$-cell in $U$. By [8] (see Theorem 3 and "added in proof"), each simplex of $K$ can be tamed in its open $n$-cell and hence is locally flat in $U$.

Corollary 4.10. If $\hat{f}$ is AFG, $n \geq 6$, and $U$ is a PL-manifold, then $U$ is bomeomorpbic to an open mapping cylinder neighborbood of $P$.

Proof. By [8], there exists a homeomorphism $e$ of $U$ onto itself such that $e(P)$ is a subpolyhedron of $U$. Let $N$ be a regular neighborhood of $e(P)$ in $U$; there exists $r>0$ such that $e b^{r} e^{-1}(N) \subseteq$ int $N$. By [36] and [40], $N-e b^{r} e^{-1}(N)$ is homeomorphic to bdry $N \times[0,1)$; hence $U-P$ is homeomorphic to bdry $N \times$ $(0,1)$ and the conclusion follows from the fact that a regular neighborhood is a closed mapping cylinder neighborhood.

Corollary 4.11. Let $U$ be an open connected $n$-manifold, $n \geq 6$, let $b$ be a bomeomorphism of $U$ onto itself sucb that $b$ is regular except at $\infty$ and on $a$ closed subset $P$ which is a polybedron of dimension $k \leq n-3, b$ is positively regular on $U$ and $b \mid P=$ identity. If the components of the link of each point in $P$ in some triangulation of $P$ are simply connected and if $\hat{f}$, the map induced on the orbit space of $b \mid U-P$, is a proper AFG map, then $P$ is locally flat in $U$.

Corollary 4.12. Let $b$ be a bomeomorphism of $S^{n}, n \geq 6$, such that $b$ is regular except on $\Sigma$, a $k$-sphere, $k \leq n-3, b$ is positively regular except at some $a \in \Sigma$, and $b \mid \Sigma$ is the identity. If $k \neq 2$ and the map induced on the orbit space of $b \mid S^{n}-\Sigma$ is AFG, then $\Sigma$ is locally flat in $U$ and bence flat.

Corollary 4.13. Let $U$ be an open connected $n$-manifold, $n \geq 6$, let $b$ be a bomeomorphism of $U$ onto itself sucb that $b$ is regular except at $\infty$ and on a com. pact manifold $P$ of dimension $k \leq n-3, k \neq 2$ ( $P$ is not necessarily triangulable), $b$ is positively regular on $U$ and $b \mid P=$ identity. If $\hat{f}$, the map induced on the orbit space of $b \mid U-P$, is AFG, then $P$ is locally flat in $U$.

\section{Necessity of AFG.}

Theorem 5.1. Let $U$ be an open connected n-manifold, let $b$ be a bomeomorphism of $U$ onto itself sucb that $b$ is regular except at $\infty$ and on a compact locally flat polybedron $P$ of dimension $k \leq n-3, n \geq 6, b$ is positively regular 
on $U$ and $b \mid P$ is the identity. Then $\hat{f}$, the map induced on the orbit space on $b \mid U-P$ to $P$, is AFG.

Proof. Let $p \in P$ and let $N_{0}$ be the open star of $p$ in $P$ for some triangulation $K$ of $P$. Consider $N=f^{-1}\left(N_{0}\right)$. We claim that $N$ is homeomorphic to Euclidean $n$-space. For let $C$ be a compactum in $N$ and let $N_{1}$ be an open $n$ cell in $N$ which contains $p$. There exists a subdivision of $K$ such that the closed star $N_{2}$ of $p$ with respect to this subdivision contains $f(C)$ in its interior. Since $P$ is locally flat, $N_{2}$ is locally flat and hence $N-N_{2}$ is 1-LC at each point of $N_{2}$. From [8], it follows that the hypotheses of Newman's engulfing theorem [31] are satisfied and hence $N_{2}$ can be engulfed by $N_{1}$. Therefore there exists an open $n$-cell $N_{3}$ in $N$ which contains $N_{1}$. By Proposition 2.1, there exists $r$ such that $b^{r}(C) \subseteq N_{3}$ and hence $C \subseteq b^{-r}\left(N_{3}\right)$. By [6], $N$ is homeomorphic to Euclidean $n$ space.

Since $N \cap P$ is locally flat in $N, \pi_{1}(N-(N \cap P))$ is trivial and $\pi_{1}\left(\hat{f}^{-1}\left(N_{0}\right)\right)$ is isomorphic to the integers.

Let $V$ be an open subset of $\hat{f}^{-1}(N)$ which contains $\hat{f}^{-1}(p)$. Let $N^{\prime}$ be the open star of $p$ in $P$ for some subdivision of $K$ such that $\hat{f}^{-1}\left(N^{\prime}\right) \subseteq V$. It is easily seen that the inclusion induces an isomorphism $\pi_{1}\left(\hat{f}^{-1}\left(N^{\prime}\right)\right) \rightarrow \pi_{1}\left(\hat{\hat{f}}^{-1}(N)\right)$ and, since there groups are Abelian, $\hat{f}$ is AFG at $p$.

6. Mapping cylinder neighborhoods. For those familiar with uv and UV properties of continua [3], [28], [29], we note that for a continuum, uv ${ }^{1}$ and AFG is equivalent to $\mathrm{UV}^{1}$. Hence from Proposition 4.1, Theorem 5.1, [8] and [27], we have the following.

Theorem 6.1. Let $g$ be a proper onto map from a locally compact metric space $Y$ to a polybedron $P$ of dimension $k$. If the open mapping cylinder of $g$ is an $n$ manifold, $n \geq 6, n \geq k+3$, then $P$ is locally flat in the open mapping cylinder of $g$ if and only if $g$ is AFG.

7. Some examples. In this section, we construct some examples of homeomorphisms with wildly embedded irregular sets.

Let $\mathrm{U}$ be a neighborhood of a subset $A$ of a space $X ; \mathrm{U}$ is an iterative neighborbood of $A$ if there exists a homeomorphism $b$ of $U-A$ onto itself such that the natural projection of $\mathrm{U}-A$ onto the orbit space $O$ is a covering map and $O$ is Hausdorff.

We recall an example due to Whitehead [39]. Let $T_{0}^{\prime} \subseteq T_{0}$ be solid tori contained in the 3-sphere as shown in Diagram 1. There exists a homeomorphism $k_{0}$ : $S^{3} \rightarrow S^{3}$ such that $k_{0}\left(T_{0}\right)=T_{0}^{\prime}$. Let $U_{0}=S^{3}, A_{0}=\bigcap_{i>0} k_{0}^{i}\left(T_{0}\right)$ and $B_{0}=$ $\mathcal{U}_{0}-\bigcup_{i<0} k_{0}^{i}\left(T_{0}\right)$.

Define inductively $\mathcal{U}_{n}$ to be the suspension of the decomposition space 


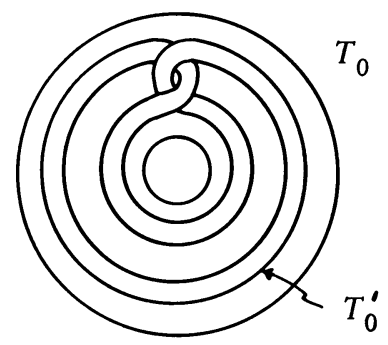

\section{Diagram 1}

$\mathrm{U}_{n-1} /\left\{A_{n-1}, B_{n-1}\right\}$ obtained from $\mathrm{U}_{n-1}$ by shrinking $A_{n-1}$ and $B_{n-1}$ to distinct points. Let $q_{n}:\left(\mathrm{U}_{n-1} /\left\{A_{n-1}, B_{n-1}\right\}\right) \times[-1,1] \rightarrow \mathrm{U}_{n}^{n-1}$ be the natural projection, $A_{n}=q_{n}\left(a_{n-1} \times[-1,1]\right)$, and $B_{n}=q_{n}\left(b_{n-1} \times 0\right)$ where $a_{n-1}$ and $b_{n-1}$ are the points in $\mathcal{U}_{n-1} /\left\{A_{n-1}, B_{n-1}\right\}$ which correspond to $A_{n-1}$ and $B_{n-1}$, respectively. (Note that $B_{n}$ is a point for $n>0$.) Let

$$
T_{n}=q_{n}\left(T_{n-1} \times[-1,1]\right) \cup\left(\mathrm{U}_{n-1} /\left\{A_{n-1}, B_{n-1}\right\}\right) \times([-1,-1 / 2] \cup[1 / 2,1])
$$

and

$$
T_{n}=q_{n}\left(T_{n-1}^{\prime} \times[-1,1]\right) \cup\left(\mathcal{U}_{n-1} /\left\{A_{n-1}, B_{n-1}\right\}\right) \times([-1,-2 / 3] \cup[2 / 3,1]) .
$$

There is a homeomorphism $k_{n}$ of $\mathcal{U}_{n}$ onto itself such that $k_{n}\left(T_{n}\right)=T_{n}^{\prime}, A_{n}=$ $\bigcap_{i>0} k_{n}^{i}\left(T_{n}\right)$, and $B_{n}=\mathcal{U}_{n}-\bigcup_{i<0} k_{n}^{i}\left(T_{n}\right)$. We have the following facts.

(i), $\mathcal{U}_{n}^{n}-B_{n}$ is an iterative neighborhood of $A_{n}$ in $\mathcal{U}_{n}$.

(ii) $\mathcal{U}_{n}$ is an $(n+3)$-sphere and $\left(\mathcal{U}_{n}-B_{n}\right) / A_{n} \times R$ is homeomorphic to $R^{n+4}$ by [30] and [2] if $n=1$ and by [1] if $n>1$.

(iii) $\pi_{1}\left(U_{n}-A_{n}\right)$ is infinitely generated and hence $A_{n}, n>0$, is a wildly embedded arc in $\mathcal{U}_{n}^{n}$ [7].

(iv) $a_{n} \times[-1,1]$ is a cellular wildly embedded arc in $\left(U_{n}-B_{n}\right) / A_{n} \times R[7]$.

Theorem 7.1. For each $n \geq 4$ and $1 \leq k \leq n-3$, there exists a bomeomorphism $b$ of the $n$-sphere $S^{n}$ onto itself, a $k$-cell $D$ wildly embedded in $S^{n}, x \in S^{n}-D$ sucb that $b$ is regular except on $D \cup\{x\}$, positively regular except on $\{x\}$ and negatively regular except on $D$. Moreover, $D$ is cellular in $S^{n}$ and if $b$ is orientation-preserving and $n \geq 6$, then $b \mid S^{n}-D$ can be embedded in a flow.

Proof. Let $B$ be the unit $k$-cell with center at the origin in $R^{k}$; each point in $R^{k}$, except the origin, can be expressed uniquely in the form $(\phi, t)$ where $\phi \epsilon$ bdry $B$, and $t>0$. Consider $R^{n}=\left(\mathcal{U}_{n-k-1}-B_{n-k-1}\right) / A_{n-k-1} \times R^{k}$ and let $D=$ $\left\{a_{n-k-1}\right\} \times B$.

Define $b: R^{n} \rightarrow R^{n}$ by 


$$
\begin{aligned}
b([x], \phi, t) & =\left(\left[b_{n-k-1}(x)\right], \phi, 1 / 2(t-1)\right), & & t \geq 1, \\
& =\left(\left[b_{n-k-1}(x)\right], \phi, t\right), & & t \leq 1, \\
b\left(a_{n-k-1}, \phi, t\right) & =\left(a_{n-k-1}, \phi, 1 / 2(t-1)\right), & & t \geq 1, \\
& =\left(a_{n-k-1}, \phi, t\right), & & t \leq 1,
\end{aligned}
$$

where $x \in \mathcal{U}_{n-k-1}-\left(A_{n-k-1} \cup B_{n-k-1}\right),[x]$ is the corresponding point in $\left(\mathrm{U}_{n-k-1}-B_{n-k-1}\right) / A_{n-k-1},(\phi, t) \in R^{k}$ and $b_{n-k-1}$ is the homeomorphism which is given in the definition of iterative neighborhood. $b$ has a unique extension to the $n$-sphere $S^{n}=R^{n} \cup\{\infty\}$.

$b$ is the desired homeomorphism. To see that $b / S^{n}-D$ can be embedded in a flow, we refer the reader to [18].

As another application of the iterative neighborhood construction, we have

Theorem 7.2. For each $n \geq 5$, there exists a closed connected n-manifold $M$, disjoint closed connected submanifolds, $N_{1}$ and $N_{2}$, of $M$, and a bomeomorphism $b$ of $M$ onto itself such that $b$ is regular except on $N_{1} \cup N_{2}$ and $M-\left(N_{1} \cup N_{2}\right)$ does not have the bomotopy type of a finite complex.

Proof. Let $M=S^{2} \times\left(\mathcal{U}_{n-2} /\left\{A_{n-2}, B_{n-2}\right\}\right), N_{1}=S^{2} \times\left[B_{n-2}\right]$ and $N_{2}=S^{2} \times$ $\left[A_{n-2}\right]$. Define $b: M \rightarrow M$ by

$$
b(s,[x])=\left(s,\left[k_{n-2}(x)\right]\right), \quad b\left(s, a_{n-2}\right)=\left(s, a_{n-2}\right), \quad b\left(s, b_{n-2}\right)=\left(s, b_{n-2}\right)
$$

for $s \in S^{2}$ and $x \in \mathrm{U}_{n-2}-\left(A_{n-2} \cup B_{n-2}\right) . b$ is the desired homeomorphism and note that $\pi_{1}\left(M-\left(N_{1} \cup N_{2}\right)\right)$ is infinitely generated.

There are also homeomorphisms of $S^{3}$ with wild irregular sets. In the next example, we modify a construction due to Kinoshita [25] and simplified in [26].

Theorem 7.3. There is a bomeomorphism $b$ on the 3-sphere $S^{3}$ and an arc $A$ in $S^{3}$ such that $b$ is regular on $S-A$, not regular on $A$ and $A$ is not tamely em. bedded in $S^{3}$.

Proof. (We express our gratitude to Professor C. Sikkema for correcting an error in an earlier example.) Let $f: S^{2} \rightarrow[0,1]$ be a map such that $f^{-1}(0)$ is a disk, $f^{-1}(1)$ is a point and $f^{-1}(c), c \in(0,1)$, is a simple closed curve. Let $M$ be the mapping cylinder of $f$ and let $\pi:\left(S^{2} \times[0,1] \cup[0,1]\right) \rightarrow M(\pi(s, 0)=\pi(f(s)))$ be the natural projection. Note that int $M$ is homeomorphic to $E^{3}$ and $\pi([0,1])$ is locally tame at each point.

Let $k: E^{1} \rightarrow(0,1)$ be an order-preserving homeomorphism and let $\lambda: E^{1} \rightarrow$ $E^{1}$ be defined by $\lambda(x)=x-1$. Define $t:$ int $M \rightarrow$ int $M$ by 


$$
\begin{aligned}
t(\pi(s, y)) & =\pi\left(s, k \lambda k^{-1}(y)\right) & & (s, y) \in S^{2} \times(0,1), \\
t(\pi(y)) & =\pi(y) & & y \in[0,1] .
\end{aligned}
$$

Note that $t$ is a homeomorphism of int $M$ onto itself.

Let $\phi:[0,1] \rightarrow f^{-1}(0) \times[k(0), k(1)]$ be an embedding such that $\phi([0,1]) \cap$ $S^{2} \times k(i), i=0,1, t(\pi(\phi(1)))=\pi(\phi(0))$, and $\phi([0,1])$ fails to be locally tame only at $\phi(1)$. If $M_{\infty}=$ int $M \cup\{\infty\}$ is the one-point compactification of int $M$, let $\alpha=$ $\{\pi(0), \infty\} \cup \bigcup_{i=-\infty}^{+\infty} t^{i} \pi \phi([0,1])$. By [9], $\alpha$ is cellular in $M_{\infty}$. Hence there is a map $g$ of $M_{\infty}$ onto itself such that $g(\alpha)=\pi(0)$ and $g \mid M_{\infty}-\alpha$ is a homeomorphism.

Define $b: M_{\infty} \rightarrow M_{\infty}$ by $b(x)=g t^{\prime} g^{-1}(x)$ where $t^{\prime}(x)=x$ for $x \in$ int $M$ and $t^{\prime}(\infty)=\infty$. Let $A=g \pi([0,1])$. Since $g^{-1}(A)=\pi([0,1]) \cup \alpha, A$ fails to be locally tame at $g \pi(0)[25] . b$ is the desired homeomorphism and $A$ is the desired arc.

Corollary 7.4. For each $n \geq 3$, there is a wild $(n-2)$-cell $D_{n-2}$ in $S^{n}$ and a bomeomorphism $b_{n}$ of $S^{n}$ sucb that $b_{n}$ is regular on $S^{n}-D_{n-2}$ but not regular on $D_{n-2}$.

Proof. The arc $A$ in Theorem 7.3 can be chosen so that $\Sigma^{k} A$ is wild in $S^{k+2}$ at the points corresponding to the suspension of $g \pi(0)$. Let $D_{1}=A, b_{3}$ the homeomorphism in Theorem 7.3, and $p_{3}=g \pi(1)$. Having chosen $b_{n-1}, D_{n-3}$, and $p_{n-1}$, let $\bar{b}_{n}=$ suspension of $b_{n-1}$ and let $\bar{D}_{n-2}$ be $\Sigma D_{n-3}$, then $\bar{b}_{n}$ satisfies our conditions except that it is regular at the suspension points. To correct this, let $B=\Sigma P_{n-1}$ and let $\rho: S^{n} \rightarrow S^{n} / B$ be the quotient map. Let $b_{n}=\rho b_{n-1} \rho^{-1}$, $D_{n-2}=\rho\left(\bar{D}_{n-2}^{n-1}\right)$, and $p_{n}=\rho\left(p_{n-1}\right)$. Then $b_{n}$ and $D_{n-2}$ satisfy the necessary conditions.

Theorem 7.5. If $A$ is a tame arc in $E^{3}$, then there exist infinitely many dis. tinct topological types of homeomorphisms on $E$ which are regular and properly discontinuous on $E-A$, are not regular on $A \cup\{\infty\}$ and are positively regular on $E^{3}$.

Proof. Suppose $b$ is a homeomorphism of $E^{3}$ and $A$ is a tame arc in $E^{3}$ such that $b$ is regular on $E^{3}-A$, not regular on $A$, positively regular on $E^{3}$ and $b \mid A$ is the identity. Note that $f^{-1}$ (bdry $A$ ) is an invariant of the topological type of $b$, where $f=\lim _{i \rightarrow+\infty} b^{i}$; i.e. if $k=\lambda b \lambda^{-1}$ and $g=\lim _{i \rightarrow+\infty} k^{i}$, then $g^{-1}$ (bdry $A$ ) is homeomorphic to $f^{-1}$ (bdry $A$ ). It is easy to define a homeomorphism $b_{n}$ of $E^{3}$ onto itself, $n=0,1,2, \ldots$, which has the desired properties listed in Theorem 7.5 and such that the components of $f_{n}^{-1}$ (bdry $A$ ), $f=\lim _{i \rightarrow+\infty} b_{n}^{i}$, are homeomorphic to $[0,+\infty) \cup \bigcup_{i=-\infty}^{+\infty} D_{i}$ where $C_{i}$ is the cone over $n$ points, $C_{i}$ $\cap C_{j}=\varnothing$ for $i \neq j, C_{i} \cap[0,+\infty)=\{x \mid x=i$ if $i>0$ and $x=1 /(2-i)$ if $i \leq 0\}$ and $\lim _{i \rightarrow-\infty} \operatorname{diam} C_{i}=0$. 
Theorem 7.6. There is a bomeomorphism $b$ of $S^{1} \times R^{2}$ onto itself which is regular on $S^{1} \times\left(R^{2}-\{0\}\right)$, is positively regular everywhere, and sucb that $\overline{\lim }_{i \rightarrow+\infty} b^{i}(x)=S^{1} \times\{0\}$ for each $x \in S^{1} \times E^{2}$.

Proof. Let $k: S^{1} \rightarrow S^{1}$ be rotation through an irrational multiple of $\pi$ radians, and let $b(x, t)=(k(x), t / 2)$. It is easily seen that $b$ satisfies the desired conditions.

Theorem 7.7. There is a bomeomorphism $b$ of $S^{3}$ onto itself which is regular except on a 1 -sphere $\Sigma$, is positively regular except at point $s \in \Sigma$ and $b \mid \Sigma$ is the identity and $\Sigma$ is not locally flat in $S^{3}$.

Proof. This example can be obtained from the example in Theorem 7.3 by shrinking a locally tame invariant arc whose boundary is $\{\infty, g \pi(1)\}$.

\section{BIBLIOGRAPHY}

1. J. J. Andrews and M. L. Curtis, n-space modulo an arc, Ann. of Math。 (2) 75 (1962), 1-7. MR $25 \# 2590$.

2. J. J. Andrews and L. Rubin, Some spaces whose product with $E^{1}$ is $E^{4}$, Bull. Amer. Math. Soc. 71 (1965), 675-677. MR 31 \#726.

3. S. Armentrout, UV properties of compact sets, Trans. Amer. Math. Soc. 143 (1969), 487-498. MR $42 \# 8451$ 。

4. K. Borsuk, Theory of retracts, Monografie Mat., Tom 44, PWN, Warsaw, 1967. MR $35 \# 7306$.

5. W. Browder, Structures on $M \times R$, Proc。 Cambridge Philos. Soc。 61 (1965), 337345. MR 30 \#5321.

6. M. Brown, The monotone union of open n-cells is an n-cell, Proc. Amer. Math. Soc. 12 (1961), 812-814. MR 23\#A4129.

7. - Wild cells and spheres in higher dimensions, Michigan Math. J. 14 (1967), 219-224. MR $36 \# 4533$.

8. J. L. Bryant and C. L. Seebeck III, Locally nice embeddings in codimension three, Quart. J. Math. Oxford (2) 21 (1970), 265-272.

9. P. H. Doyle and J. Neufeld, A sufficient condition that an arc in $S^{n}$ be cellular, Pacific J. Math. 14 (1964), 501-503; correction, ibid. 15 (1965), 1474. MR 28 \#5429; MR $32 \# 3051$.

10. P. F. Duvall, Jr. and L. S. Husch, Analysis on topological manifolds, Fund. Math. 77 (1972), 75-90.

11. - Regular properly discontinuous $Z^{n}$-actions on open manifolds, Illinois J. Math. (to appear).

12. S. Eilenberg and N. E. Steenrod, Foundations of algebraic topology, Princeton Univ. Press, Princeton, N. J., 1952. MR 14, 398.

13. H. Freudenthal, Neuaufbau der Endentheories, Ann. of Math。 (2) 43 (1942), 261279. MR 3, 315.

14. O. G. Harrold, Jro, Euclidean domains with uniformly Abelian local fundamental groups, Trans. Amer。 Math. Soc。67 (1949), 120-129. MR 11, 381.

15. T. Homma and S. Kinoshita, On a topological characterization of the dilatation in $E^{3}$, Osaka Math. J. 6 (1954), 135-143. MR 16, 160 .

16. - On homeomorphisms which are regular except for a finite number of points, Osaka Math.J. 7 (1955), 29-38. MR 16, 1140.

17. J. F. P. Hudson, Piecewise linear topology, Benjamin, New York, 1969. MR $40 \# 2094$. 
18. L. S. Husch, A homotopy theoretic characterization of the translation in $E^{n}$, Compositio Math. 24 (1972), 55-61.

19. - Topological characterization of the dilation and the translation in Frechet spaces, Math. Ann. 190 (1970/71), 1-5. MR 43 \#8068.

20. - A topological characterization of the dilation in $E^{n}$, Proc. Amer. Math. Soc. 28 (1971), 234-236. MR 44 \#1051.

21. S. K. Kaul, On almost regular homeomorphisms, Canad. J. Math. 20 (1968), 1-6. MR $36 \# 5908$.

22. B. V. Kerékjártó; Topologische characterisierungen der linearen Abbildungen, Acta Litt. Acad. Sci. Szeged 6 (1934), 235-262.

23. S. Kinoshita, Notes on covering transformation groups, Proc. Amer. Math. Soc. 19 (1968), 421-424. MR 36 \#5921.

24. - On quasi translations in 3-space, Topology of 3-Manifolds and Related Topics (Proc. Univ. of Georgia Inst., 1961), Prentice-Hall, Englewood Cliffs, N. J., 1962, pp. 223-226. MR $25 \# 3116$.

25. - On quasi-translations in 3-space, Fund. Math. 56 (1964), 69-79. MR 30 \#1502.

26. S. Kinoshita and C. D. Sikkema, On arc and ball pairs and quasi-translations of the 3-sphere, Michigan Math. J. 17 (1970), 165-173. MR 41 \#4511.

27. R. C. Lacher, Cell-like mappings. II, Pacific J. Math. 35 (1970), 649-660. MR $43 \# 6936$.

28. - Cell-like spaces, Proc.Amer. Math. Soc. 20 (1969), 598-602.

MR 38 \#2754.

29. R. C. Lacher and D. R. McMillan, Jr., Partially acyclic mappings between manifolds, Amer. J. Math. 94 (1972), 246-266.

30. D. R. McMillan, Jr., Cartesian products of contractible open manifolds, Bull. Amer. Math. Soc. 67 (1961), 510-514. MR 24 \#A1132.

31. M. H. A. Newman, The engulfing theorem for topological manifolds, Ann. of Math. (2) 84 (1966), 555-571. MR 34 \#3557.

32. J.-P. Serre, Homologie singulière des espaces fibrés. Applications, Ann. of Math. (2) 54 (1951), 425-505. MR 13, 574.

33. L. C. Siebenmann, The obstruction to finding a boundary for an open manifold of dimension greater than five, Thesis, Princeton University, Princeton, N. J., 1965.

34. - On detecting open collars, Trans. Amer. Math. Soc. 142 (1969), 201227. MR 39 \#605.

35. E. H. Spanier, Algebraic topology, McGraw-Hill, New York, 1966. MR 35 \#1007.

36. J. Stallings, On infinite processes leading to differentiability in the complement of a point, Differential and Combinatorial Topology (A Sympos. in Honor of Marston Morse), Princeton Univ. Press, Princeton, N. J., 1965. pp. 245-254. MR 31 \#5213.

37. C. T. C. Wall, Finiteness conditions for CW-complexes, Ann. of Math. (2) 81 (1965), 56-69. MR $30 \# 1515$. $244-245$.

38. - Surgery on compact manifolds, Academic Press, New York, 1970, Pp.

39. J. H. C. Whitehead, A certain open manifold whose group is unity, Quart. J. Math. Oxford 6 (1935), 364-366.

40. E. C. Zeeman, Seminar on combinatorial topology, Inst. Hautes Études Sci., Paris, 1963.

41. P. F. Duvall, Jr. and L. S. Husch, Taming irregular sets of homeomorphisms, Bull. Amer. Math. Soc. 78 (1972), 77-79.

DEPARTMENT OF MATHEMATICS, VIRGINIA POLYTECHNIC INSTITUTE AND STATE UNIVERSITY, BLACKSBURG, VIRGINIA 24601

DEPARTMENT OF MATHEMATICS, OKLAHOMA STATE UNIVERSITY, STILLWATER, OKLAHOMA 74074 (Current address of P. F. Duvall, Jr.)

DEPARTMENT OF MATHEMATICS, UNIVERSITY OF TENNESSEE, KNOXVILLE, TENNESSEE 37916 (Current address of L. S. Husch) 